\title{
Factores organizacionales que influyen en el estrés laboral del personal docente en universidades del Área Metropolitana de Monterrey
}

\author{
César Perales Martínez y María de Jesús Araiza Vázquez²

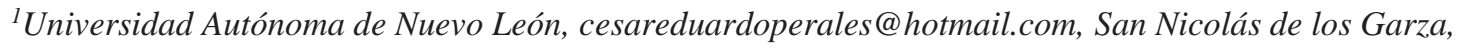 \\ Nuevo León, México, 8211119961. \\ ${ }^{2}$ Universidad Autónoma de Nuevo León, araizav@gmail.com, San Nicolás de los Garza, Nuevo León, \\ México, 8113116160.
}

\author{
Información del artículo revisado por pares \\ Fecha de aceptación: junio-2021 \\ Fecha de publicación en línea: diciembre-2021 \\ DOI: https://doi.org/10.29105/vtga7.2-34
}

\section{Resumen}

Tener un trabajo remunerado es, para la mayoría de las personas, una fuente de seguridad económica, estatus, bienestar y salud. Da sentido a sus vidas, pero en ocasiones puede ser una carga física $y / o$ mental, y una fuente de frustración, conflicto, decepción, malestares físicos y mentales, e incluso la muerte (Lundberg $\&$ Cooper, 2011). Estos malestares son a veces referidos como presión laboral, o mejor conocidos como estrés laboral (Wainwright \& Calnan, 2002). El propósito de este estudio es hacer una revisión crítica de la literatura para integrar un marco teórico y establecer tanto hipótesis general como hipótesis específicas y los factores que, a partir de la revisión de la teoría y estudios empíricos, se postulan como influyentes sobre la variable dependiente del estudio. Además, se reporta el instrumento a utilizar en una futura fase de investigación, en donde se cuantificarán las relaciones entre las variables propuestas.

Palabras clave: educación superior, estrés laboral, docentes.

\begin{abstract}
Having a paid job is, for most people's work, a source of economic security, status, well-being and health. It gives meaning to their lives, but at times it can be a physical and / or mental burden, and a source of frustration, conflict, disappointment, physical and mental discomfort, and even death (Lundberg \& Cooper, 2011). These discomforts are sometimes referred to as job pressure, or better known as job stress (Wainwright \& Calnan, 2002). The purpose of this study is to make a critical review of the literature to integrate a theoretical framework and establish both general hypotheses and specific hypotheses and the factors that, from the review of the theory and empirical studies, are postulated as influencing the dependent study variable. In addition, the instrument to be used in a future research phase is reported, where the relationships between the proposed variables will be quantified.
\end{abstract}

Keywords: higher education, job stress, academics. JEL: I12, I23, J81. 


\section{INTRODUCCIÓN}

Los orígenes del estrés laboral pueden ser ubicados en varios puntos históricos alrededor del mundo. La revolución industrial y las guerras mundiales, por ejemplo, son algunos hitos históricos que han cambiado la forma en que se trabaja y se percibe el trabajo. A partir de la Revolución Industrial, originada en algunos países europeos, se logró un gran crecimiento económico y tecnológico, lo que ocasionó cambios significativos en la forma de trabajar de la fuerza laboral, que a su vez provocaron el desarrollo de políticas de asistencia social, incluida la legislación para propiciar la salud y seguridad de la fuerza laboral (Siegrist, 2016).A partir de segundo párrafo utilizar sangría en primera línea.

El estrés laboral como tal no había sido formulado formalmente en la primeras décadas del siglo XX pero para la década de 1930 ya se investigaba el estrés en algunas instituciones del sector servicio, y es que algunos científicos sugieren que es muy complejo trabajar en este sector (Dollard, Winefield, \& Winefield, 2003). Por ejemplo en el sector educativo, en 1933 se llevó a cabo uno de los primeros estudios sobre la ocurrencia del estrés en maestros (Humphrey, 1998). Se desarrolló un estudio en el que participaron 600 maestros, en el que se reveló que $17 \%$ estaban "inusualmente nerviosos" y que un $11 \%$ habían sufrido crisis nerviosas. Esto sirvió de impulso a muchos otros restudios del estrés laboral en el sector educativo durante las décadas siguientes.

La primera encuesta destinada a evaluar el estrés laboral en diferentes ocupaciones se llevó a cabo en 1985 en el Reino Unido, comisionada por The Sunday Times en la que se evaluaron 100 trabajos en una escala de 10 puntos, con 1 siendo lo menos estresante hasta 10 siendo lo más estresante (Cranwell-Ward \& Abbey, 2005).

El problema del estrés laboral se ha acentuado tanto en los últimos años que en México se comenzaron poner en marcha acciones legales para lidiar con este problema. La Secretaría de Trabajo y Prevención Social presentó ante el Comité Consultivo Nacional de Normalización y Seguridad y Salud en el
Trabajo, el 26 de septiembre de 2016, el Proyecto Norma Oficial Mexicana PROYNOM-035-STPS-2016, Factores de riesgo psicosocial-identificación y prevención (DOF, 2018). La NOM 035 entró en vigor en dos etapas, las cuales delimitaron los requisitos con los que los centros de trabajo tendrán que dar cumplimiento:

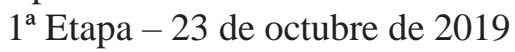

La política; las medidas de prevención; la identificación de los trabajadores expuestos a acontecimientos traumáticos severos, y la difusión de la información.

$2^{\text {a }}$ Etapa -23 de octubre de 2020

La identificación y análisis de los factores de riesgo psicosocial; la evaluación del entorno organizacional; las medidas y acciones de control; la práctica de exámenes médicos, y los registros.

La importancia de este estudio en el contexto de la educación superior es que las universidades tienen un rol de gran importancia en la economía y vida social de las naciones, ya que gracias a las universidades se producen los ingenieros, doctores, científicos y otros profesionales, los cuales producen la mayoría de las investigaciones innovadoras (Dollard, Winefield, \& Winefield, 2003). Sin embargo, desde hace más de veinte años la capacidad de las universidades para seguir produciendo ese capital humano tan importante ha sido amenazada con el deterioro de condiciones laborales, como menciona Shirley Fisher (1994, p. 61): "Las exigencias a académicos se han elevado con rapidez en los últimos diez años... ha habido una erosión en el control del trabajo. Todas las señales apuntan a que este problema continuará".

Según la literatura del estrés laboral en personal académico, los factores que suelen causar estrés laboral en personal académico son los siguientes: sobrecarga de trabajo (Guillespie, Walsh, Winefield, Dua, \& Stough, 2001), (Kinman, 2001), (Idris, 2011) (Bowling, Alarcon, Bragg, \& Hartman, 2015); inseguridad laboral (Mudrak, y otros, 2017) (Winefield, Boyd, Seabel, \& Pignata, 2008); injusticia en procedimientos (Boyd, y otros, 2011) (Judge \& Colquitt, 2004) (Wainwright \& Calnan, 2002); recompensas insuficientes (Tacca-Huanmán \& Tacca-Huanmán, 2019) 
(Kinman, 2016) (Guillespie, Walsh, Winefield, Dua, \& Stough, 2001) y conflicto trabajo-familia (Kinman \& Jones, 2003) (Bell, Rajendran, \& Theiler, 2010) (Panatik, y otros, 2012).

Con base en las evidencias señaladas anteriormente se plantea la siguiente pregunta de investigación:

¿Qué factores organizacionales influyen en el estrés laboral del personal docente de universidades del área metropolitana de Monterrey?

\section{MARCO TEÓRICO}

En este apartado se ofrece la sustentación teórica del problema de investigación. Es decir, se presentan las teorías que relacionan los elementos básicos de la investigación, que son: las variables de investigación, la forma en que se relacionan y el porqué de estas relaciones.

\subsection{Definiciones}

A continuación, se presentan las definiciones formales de cada una de las variables del estudio (Véase tabla 1).

Tabla 1 Definiciones de variables

\begin{tabular}{|l|l|}
\hline Concepto & Definición \\
\hline $\begin{array}{l}\text { Sobrecarga de } \\
\text { trabajo }\end{array}$ & $\begin{array}{l}\text { Cantidad y dificultad del trabajo a } \\
\text { desempeñar (Bowling \& } \\
\text { Kirkendall, 2012) }\end{array}$ \\
\hline $\begin{array}{l}\text { Inseguridad } \\
\text { laboral }\end{array}$ & $\begin{array}{l}\text { Preocupación en relación al } \\
\text { futuro de la situación laboral, ya } \\
\text { sea perder el trabajo en su } \\
\text { totalidad o características } \\
\text { valiosas del trabajo (Hellgren et } \\
\text { al., 1999) }\end{array}$ \\
\hline $\begin{array}{l}\text { Injusticia } \\
\text { procedimental }\end{array}$ & $\begin{array}{l}\text { Es la percepción de escaza } \\
\text { justicia y equidad en los procesos } \\
\text { por los cuales las decisiones } \\
\text { organizacionales son hechas y } \\
\text { las recompensas son } \\
\text { determinadas (Cohen \& Spector, } \\
\text { 2001) }\end{array}$ \\
\hline $\begin{array}{l}\text { Recompensas } \\
\text { insuficientes }\end{array}$ & $\begin{array}{l}\text { Percepciones que tienen los } \\
\text { individuos respecto a la } \\
\text { desigualdad entre los estímulos } \\
\text { financieros y no financieros que } \\
\text { reciben a cambio de su trabajo } \\
\text { (Armstrong M., 2009) }\end{array}$ \\
\hline $\begin{array}{l}\text { Conflicto } \\
\text { trabajo-familia }\end{array}$ \\
obligaciones del trabajo complica \\
\hline
\end{tabular}

\begin{tabular}{|l|l|}
\hline & $\begin{array}{l}\text { cumplir con las obligaciones con } \\
\text { la familia (Greengaus \& Beutell, } \\
1985)\end{array}$ \\
\hline Estrés laboral & $\begin{array}{l}\text { Conjunto de reacciones que } \\
\text { suceden cuando as capacidades } \\
\text { de un individuo no pueden lidiar } \\
\text { con la presión del trabajo (HSC, } \\
\text { 1999). }\end{array}$ \\
\hline
\end{tabular}

\subsection{Modelos teóricos del estrés}

El estrés laboral puede ser explicado por varios modelos teóricos que se dividen en dos categorías principales: tempranas y contemporáneas. Los modelos tempranos se dividen en los modelos basados en el estímulo y en la respuesta. Los modelos contemporáneos se dividen en los modelos interaccionales y transaccionales (Gráfica 1).

Figura 1 Definiciones de variables

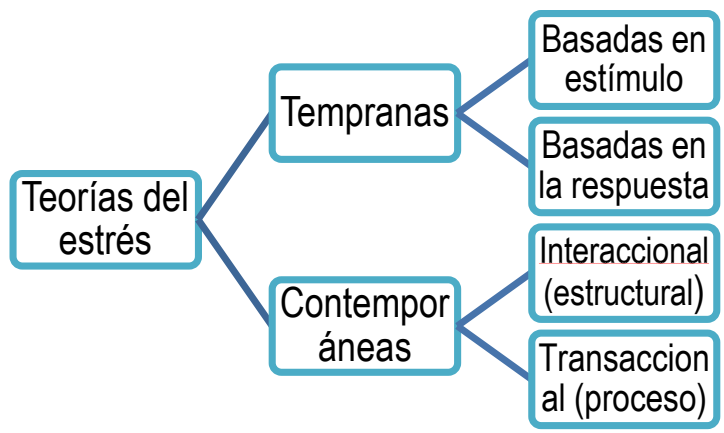

Fuente: Elaboración propia.

Cuando se buscan alternativas para conceptualizar al estrés, el resultado es un conjunto de términos que entran en la categoría de "respuesta" como: estar estresado, tensión o presión (Weinberg, Sutherland, \& Cooper, 2010). Entonces se puede inferir que, si se desea entender el concepto de estrés, se observará como una consecuencia. Por el contrario, el modelo de estrés como estímulo implica que el estrés son aquellos elementos del entorno que ejercen presión sobre el individuo.

Los modelos interaccional $y$ transaccional del estrés corresponden a las teorías contemporáneas del estrés laboral que surgieron en la década de 1970 y son 
caracterizadas por la interacción entre el individuo y el ambiente, además de que reconocen la importancia del rol activo de la persona, lo que introdujo una serie de conceptos psicológicos a la teoría del estrés (Cox \& Griffiths, 2010).

Estos modelos contemporáneos definen al estrés como una experiencia negativa que sufren los individuos cuando son sujetos a demandas excesivas con las que no pueden lidiar. Los modelos interaccional $\mathrm{y}$ transaccional se diferencian en cuán activo consideran al individuo en determinar las situaciones estresantes y sus resultados. Las teorías interaccionales se enfocan en la configuración de las situaciones, mientras que las teorías transaccionales se enfocan en las dinámicas internas del individuo como la valoración y el afrontamiento.

De los modelos descritos anteriormente, el interaccional es el que ha generado las teorías más relevantes en el estudio del estrés (desde la perspectiva de la administración).

Todas las teorías sobre el estrés comparten el elemento de la exigencia o demanda y sugieren que cuando una persona considera que la magnitud de la carga de trabajo es mayor a otros elementos como el control, las recompensas, los recursos o el clima de trabajo saludable, se genera el estrés laboral (Tabla 2).

Tabla 2 Definiciones de variables

\begin{tabular}{|l|l|}
\hline Teoría & Se refiere a: \\
\hline $\begin{array}{l}\text { Job Demand - } \\
\text { Control }\end{array}$ & $\begin{array}{l}\text { Muchas demandas laborales y } \\
\text { poco control provocan el } \\
\text { estrés. }\end{array}$ \\
\hline $\begin{array}{l}\text { Effort-Reward } \\
\text { Imbalance }\end{array}$ & $\begin{array}{l}\text { Muchas demandas y pocas } \\
\text { recompensas provocan estrés }\end{array}$ \\
\hline $\begin{array}{l}\text { Job Demands- } \\
\text { Resources Model }\end{array}$ & $\begin{array}{l}\text { Recursos disponibles actúan } \\
\text { como paliativo de las } \\
\text { demandas laborales }\end{array}$ \\
\hline $\begin{array}{l}\text { Psychological } \\
\text { Safety Climate }\end{array}$ & $\begin{array}{l}\text { Procurar la seguridad } \\
\text { psicológica disminuye el } \\
\text { estrés. }\end{array}$ \\
\hline
\end{tabular}

Fuente: Elaboración propia.

La razón por la cual se propone investigar junto al estrés laboral las variables independientes listadas en la tabla 1 es por la estrecha relación que tienen con las teorías interaccionales del estrés. Por ejemplo, la sobrecarga de trabajo y el conflicto trabajofamilia pueden ser vistos como una demanda en la teoría de Karasek, como un esfuerzo en la teoría de Siegrist y como una exigencia en la teoría de Bakker \& Demerouti. Y se espera que, si estas variables presentan mediciones altas, también lo sean las mediciones del estrés laboral.

La justicia procedimental, la seguridad laboral y las recompensas puede ser vista como un recurso en el modelo de Baker \& Demerouti el cuál puede ser usado por el individuo para sobrellevar las exigencias de su trabajo. Como estos son los recursos con los que se puede hacer frente al estrés, si se obtienen valores bajos al medir estas variables, se espera que los niveles de estrés laboral sean altos.

\section{MÉTODO}

\subsection{Participantes}

Se planea obtener los datos vía un cuestionario online. Se enviará una invitación a participar al personal académico de las universidades del área metropolitana de Monterrey, Nuevo León.

\subsection{Instrumento de medición}

Para medir todas las variables del estudio se usarán 6 cuestionarios con escala Likert de 5 puntos. Para el caso de la variable dependiente estrés laboral, se optó por la versión de 12 ítems del Cuestionario de Salud General (GHQ por sus siglas en inglés) desarrollado por Goldberg \& Williams (1988). Ejemplos de los ítems de este instrumento son: “ ¿Ha podido concentrarse en lo que hace?”, “¿Ha tenido dificultades para tomar decisiones?"

Para el caso de la variable sobrecarga de trabajo se extrajo una sección del Cuestionario Psicosocial de Copenhagen desarrollado en el departamento de psicología del Instituto Nacional de Salud Laboral Copenhagen de Dinamarca, que consta de 8 ítems para medir la sobrecarga cuantitativa y cualitativa del trabajo. Ejemplos de ítems son: “¿Tiene que trabajar muy rápido?”, “Su trabajo requiere de mucho conocimiento?"

Para la variable inseguridad laboral se eligió la Escala de Inseguridad Laboral de Hellgren et al. (1999), que consta de 6 ítems 
para medir las dimensiones cuantitativa y cualitativa de la variable. Ejemplos de ítems son: "Me preocupa perder mi trabajo antes de lo que me gustaría", "Mis oportunidades laborales futuras en esta institución son desfavorables".

La injusticia procedimental será medida con la dimensión de procedimientos formales del instrumento Escala de Justicia de Niehoff \& Moorman (1993), que consta de 6 ítems. Ejemplos de ítems son: "Mis superiores toman decisiones de manera imparcial", "Mis superiores escuchan a todo el personal antes de tomar una decisión".

La variable conflicto trabajo-familia será medida con 4 ítems desarrollados por Voydanoff (2007). Ejemplos de ítems son: "¿Qué tan seguido le ha faltado el tiempo para convivir con personas importantes en tu vida a causa del trabajo?”, “QQué tan seguido le han faltado energías para convivir con las personas importantes en tu vidaa causa del trabajo?".

Finalmente, para la variable recompensas, se usará la dimensión recompensa del instrumento Desequilibrio entre Esfuerzos y Recompensas desarrollado por Siegrist et al. (2007), que se divide en las dimensiones promoción laboral y reconocimiento. Ejemplos de ítems son: "Recibo el respeto que merezco de mis superiores", "Soy tratado justamente en el trabajo"

\section{RESULTADOS}

La evidencia empírica y los modelos teóricos apuntan a que considerar las variables independientes del estudio (sobrecarga de trabajo, inseguridad laboral, injusticia procedimental, recompensas insuficientes y conflicto-trabajo familia) como factores causales del estrés laboral parece ser algo congruente. La razón de esto es que las variables de investigación coinciden con los elementos teóricos de los modelos interaccionales del estrés para la explicación del estrés laboral. Y se elegirán los modelos interaccionales ya que forman parte de la categoría contemporánea de las teorías del estrés, que nacieron de la unificación de las teorías tempranas para poder explicar y predecir mejor el estrés.
Con todo lo anterior se presenta la siguiente hipótesis general de investigación: Los factores organizacionales que influyen en el estrés laboral del personal docente en universidades del área metropolitana de Monterrey son: la carga de trabajo, la inseguridad laboral, la injusticia procedimental, las recompensas insuficientes y el conflicto trabajo-familia (Gráfica 1).



\section{CONCLUSIONES}

Esta investigación nace de la necesidad de lidiar con un problema que ha crecido en los últimos años, que es la presencia del estrés laboral en los espacios de trabajo en México, en especial en el contexto de la educación superior. Para esto se toma como objetivo principal puntualizar los factores que propician la aparición del estrés laboral en los docentes de universidades del área metropolitana de Monterrey.

Para llegar al modelo de estudio propuesto, que es parte importante del objetivo de la investigación, se tuvo que revisar la literatura disponible hasta el momento. De aquí se obtuvieron los modelos teóricos más adecuados y los factores organizacionales que en teoría influyen sobre el estrés laboral en el personal docente, que son: sobrecarga de trabajo, conflicto trabajo-familia, injusticia procedimental, inseguridad laboral y recompensas insuficientes. La razón de que se eligieran estas variables es su relación estrecha con las teorías que explican el estrés laboral. Y es por este modelo que se plantearon las hipótesis de que cada factor influye sobre el estrés laboral experimentado por el personal docente en universidades del área metropolitana de Monterrey.

Este marco de trabajo servirá para la siguiente fase del estudio donde se reportarán 
las interacciones entre las variables independientes con el estrés laboral. Para poder analizar estas interacciones se hará uso del instrumento de medición que se construyó en base a 6 instrumentos diferentes que miden cada uno una variable específica del estudio. 


\section{REFERENCIAS}

Abbott, A. (2001). Chaos of Disciplines. The University of Chicago Press.

Abouserie, R. (1996). Stress, coping strategies and job satisfaction in university academic staff. Educational Psychology, 16, 49-56.

Baker, R. (1985). The study of stress at work. Annual Review of Public Health, 6, 367-381.

Bass, B., \& Bass, R. (2008). The bass handbook of leadership: Theory, research \& managerial applications. New York: The Free Press.

Bell, A. S., Rajendran, D., \& Theiler, S. (2010). Job stress, Wellbeing, Work-life balance and Work-life Conflict Among Australian Academics. Electronic Jouornal of Applied Psychology, 8(1), 25-37.

Bowling, N. A., \& Kirkendall, C. (2012). Workload: A Review of Causes, Consequences, and Potential Interventions. Contemporary Occupational Health, 221-238.

Bowling, N. A., Alarcon, G. M., Bragg, C. B., \& Hartman, M. J. (2015). A meta-analytic examination of the potential correlates and consequences of workload. Work \& Stress, 1-19.

Boyd, C. M., Bakker, A. B., Pignata , S., Winefield, A. H., Gillespie, N., \& Stough, C. (2011). A longitudinal test of the job demands-resources model among Australian university academics. Applied Psychology: An International Review, 60, 112-140.

Cox, T., \& Griffiths, A. (2010). Work-Related Stress. A Theoretical Perspective. En S. Leka, \& J. Houdmont, Occupational health psychology (págs. 31-56). Oxford: Blackwell Publishing Ltd.

Cranwell-Ward, J., \& Abbey, A. (2005). Organizational Stress. Wales: PALGRAVE MACMILLAN.

Cross, G., \& Carroll, D. (1990). Goodwill under stress: morale in UK universities. London: Association of University Teachers.

Darabi, M., Macaskill, A., \& Reidy , L. (2016). Stress among UK academics: identifying who copes best. Journal of Further and Higher Education, 41(3), 393-412.

Dicke, T., Parker, P. D., Marsh, H. W., Kunter, M., Shcmeck, A., \& Leutner, D. (20114). Selfefficacy in clasrrom management, cassroom disturbances, and emotional exhaustion: A moderated mediation analysis of teacher candidates. Journal of Educational Psychology, 106, 569-583.

DOF. (2018). NOM-035-STPS. Ciudad de México.

Dollard, M. F., Winefield, A. H., \& Winefield, H. R. (2003). Occupational Stress in the Service Professions. London: Taylor \& Francis Group.

Early, P. (1994). Lecturer's workload and factors affecting stress levels. Slough: NFER.

Gallup. (2014). State of America's schools: The path to winning againin education. Washington, DC: Gallup.

Goodell, H., Wolf, S., \& Rogers, F. B. (1986). Historical Perspective, Chapter 2. En S. Wolf, \& A. J. Fineston, Ocupational Stress. Health and Performance at Work. Littleton: MA: PSG Inc.

Guillespie, N. A., Walsh, M., Winefield, A. H., Dua, J., \& Stough, C. (2001). Occupational stress in universities: Staff perceptions of the causes, consequences and moderators of stress. Work and Stress, 15, 53-72.

Hakanen, J. J., Bakker, A. B., \& Schaufeli, W. B. (2006). Burnout and work engagement among teachers. Journal of School Psychology, 43, 495-513.

Heranshaw, L. S. (1987). The shaping of modern psychology. London: Routledge and Kegan Paul. Humphrey, J. H. (1998). Job Stress. Upper Saddle River: Pearson Education.

Idris, M. K. (2011). Over time effects of role stress on psychologycal stress among malasian public university academics. International Journal of Business and Social Science, 154-161.

ILO. (2016). Workplace Stress: A collective challenge. Turin: International Training Centre.

Johnson, S., Cooper, C., Cartwright, S., Donald, I., Taylor, P., \& Millet, C. (2005). The experience of work-related stress across occupations. Journal of Managerial Psychology, 20(2), 178187. 
Judge, T. A., \& Colquitt, A. (2004). Organizational justice and stress: the mediating role of workfamily conflict. Journal of applied psychology, 395-404.

Kahn, R. L., \& French , J. P. (1962). A summary and some tentative conclusions. Journal of Social Issues, (18), 122-127.

Khan, R. L. (1970). Some propositions towards a researchable conceptualization of stress. Social and Psychological Factors in Stress, 97-103.

Kinman, G. (2001). Pressure points: A review of research on stressors and strains in UK academics. Educational Psychology, 21, 473-492.

Kinman, G. (2016). Effort-reward imbalance and overcommitment in IK academics. Journal of Higher Education Policy and Management, 1-16.

Kinman, G., \& Jones, F. (2003). 'Running up the escalator': Stressors and strains in UK academics. Quality in Higher Education, 9(1), 21-38.

LePine, M. A., Zhang, Y., Crawford , E. R., \& Rich, B. L. (2016). Turning their pain to gain: Charismatic leader influence on follower stress appraisal and job performance. Academy of Management Journal, 59(3), 1036-1059.

Lundberg, U., \& Cooper, C. (2011). The science of occupational health: stress, psychobiology and the new world of work. Chichester, United Kingdom: Wiley-Blackwell.

Mudrak, J., Zabrodska, K., Kveton, P., Jelinek, M., Blatny, M., Solcova, I., \& Machovcova, K. (2017). Occupational Well-Being Among University Faculty: A Job-Demands Resources Model. Research in Higher Education, 1-24.

Narayanan, L., Menon, S., \& Spector, P. E. (1999). Stress in the workplace: a comparison of gender and occupations. Journal of Organizational Behavior, 20(1), 63-73.

Newton, T. (1995). "Managing Stress: Emotion and Power at Work. London: Sage.

OIT. (5 de Marzo de 2020). Historia de la OIT. Obtenido de Orgnización Internacional del Trabajo: https://www.ilo.org/global/about-the-ilo/history/lang--es/index.htm

Panatik, S. A., Rajab, A., Shah, I. M., Rahman, H. A., Yusoff, R. M., \& Badri, S. B. (2012). Workfamily conflict, stress and psycholgical strain in higher education. International Conference on Education and Management Inovation, 30, 67-71.

Peiró, J. M., \& Rodríquez, I. (2008). Estrés laboral, liderazgo y salud organizacional. Papeles del psicólogo, 68-82.

Pettit, L. (21 de Octubre de 2003). "Who's the most stressed of all?," . Obtenido de Personnel Today.

Robertson, I., \& Cooper, C. L. (2004). Premiership of pressure - Britain's most stressful jobs. Obtenido de Robertson Cooper Limited: www.robertsoncooper.com/pdf/stressfuljobs

Roeser, R. W., Schonert-Reichl, K., Jha, A., Cullen, M., Wallace, L., \& Wilensky, R. (2013). Mindfulness training and reductions in teacher stress and burnout: Results from two randomized, waitlist-control field trials. Journal of Educational Psychology, 105, 787-801.

Sernicharo, M. G., \& Chávez, M. M. (2020). El empleo en México. 2012-108. Pluralidad y Consenso, 8(37), 28-45.

Siegrist, J. (2016). Work stress and health in a globalizaed economy. The model of Effort-Reward Imbalance. Springer.

STPS. (25 de Octubre de 2008). Estadísticas del sector. Obtenido de Secretaría del Trabajo y Prevención Social: https://www.inegi.org.mx/contenidos/programas/enesccom/doc/Estadisticas\%20Laborales.p df

Stranks, J. (2005). Stress at Work. Oxford: Elsevier Butterworth-Heinemann.

Tacca-Huanmán, D. R., \& Tacca-Huanmán, A. L. (2019). Factores de riesgo psicosociales y estrés percibido en docentes universitarios. Propósitos y Representaciones, 7(3), 323-353.

Vong, L. T.-N., Ngan, H. F., \& Lo, P. C.-P. (2018). Does organizational climate moderate de relationship between job stress and intent to stay? Journal of Chinese Human Resources Management, 9(1), 2-20. 
Wainwright, D., \& Calnan, M. (2002). Work stress. The making of a modern epidemic. Philadelphia: Open University Press.

Weinberg, A., Sutherland, V. J., \& Cooper, C. (2010). Organizational stress management. A strategic aproach. New York: Palgrave Macmillan.

Winefield, A. H., Boyd, C., Seabel, J., \& Pignata, S. (2008). Update on a National University Stress Study. Australian Universities Review, 50, 20-29.

Winefield, A. H., Guillespie, N. A., Stough, C. K., Dua, J. K., \& Hapuarachchi, J. R. (2002). Occupational stress in Australian Universities. A national survey 2002. Melbourne, Australia: National Tertiary Education Union Publication. 